\title{
Venous thoracic outlet syndrome
}

\author{
Peiman Habibollahi, Dianbo Zhang, Marcin K. Kolber, Anil K. Pillai \\ Division of Vascular and Interventional Radiology, Department of Radiology, University of Texas Southwestern Medical Center, Dallas, TX, USA \\ Contributions: (I) Conception and design: P Habibollahi; (II) Administrative support: None; (III) Provision of study materials or patients: All authors; \\ (IV) Collection and assembly of data: P Habibollahi; (V) Data analysis and interpretation: None; (VI) Manuscript writing: All authors; (VII) Final \\ approval of manuscript: All authors. \\ Correspondence to: Peiman Habibollahi, MD. Assistant Professor of Vascular and Interventional Radiology, University of Texas Southwestern Medical \\ Center, Dallas, TX, USA. Email: Peiman.habibollahi@utsouthwestern.edu.
}

\begin{abstract}
Venous thoracic outlet syndrome (vTOS) is a spectrum of disease caused by external compression of the subclavian vein as it passes through the costoclavicular space. Paget-Schroetter's Syndrome (PSS) or effort thrombosis is a subtype of vTOS where compression and microtrauma to subclavian vein from repetitive arm movements results in venous thrombosis. PSS or effort thrombosis mostly affects young otherwise healthy active individuals, and this further highlights the importance of this condition. Early diagnosis and aggressive early intervention aimed at complete resolution of acute symptoms and minimizing the risk of recurrence is ultimately important and increases the likelihood of the full restoration of limb function. Several noninvasive imaging techniques are currently available to confirm the initial diagnosis including Doppler ultrasound, contrast-enhanced computed tomography, and magnetic resonance imaging. Following diagnosis, multiple algorithms exist for the management of PSS and almost all require a multidisciplinary approach. Like any other condition involving the thrombosis of deep venous system, initial step in the management is anticoagulation. Catheter-directed therapies (CDT) have also a pivotal role as the initial treatment to resolve the acute thrombosis and establish venous patency. CDT combined with medical anticoagulation and surgical decompression are the components of most treatment algorithms for the management of patients suffering from PSS.
\end{abstract}

Keywords: Thoracic outlet syndrome; catheter-directed thrombolysis (CDT); deep venous thrombosis

Submitted Feb 06, 2020. Accepted for publication Jun 04, 2020.

doi: $10.21037 / \mathrm{cdt}-20-168$

View this article at: http://dx.doi.org/10.21037/cdt-20-168

\section{Introduction}

Upper extremity central venous thrombosis is an increasingly common condition secondary to a wide variety of differential diagnosis $(1,2)$. Venous thoracic outlet syndrome (vTOS) or extrinsic subclavian vein compression is one of the welldefined diagnoses for this condition. First described by Sir James Paget in 1875 and later correctly attributed to venous thrombosis by Von Schroetter in 1884 (2,3), the term PagetSchroetter's Syndrome (PSS) was introduced about 70 years later by Hughes in 1949 in a report describing a large series of patients with this condition (4).

vTOS reflects a spectrum of disease secondary to anatomical narrowing in the costoclavicular triangle, which may result in thrombosis of the subclavian-axillary veins causing acute symptoms such as pain and upper extremity swelling. Although, the terms "vTOS" and "PSS" are often used interchangeably, PSS or effort-related thrombosis is used in literature to describe a subtype of vTOS patients in whom the narrowing of the costoclavicular triangle alone with repeated microtrauma from upper extremity provocative movements and subclavian vein compressiondecompression results in the venous thrombosis and occlusion (5). In contrast, McCleery syndrome is an eponym used to describe intermittent compression of the subclavian vein within costoclavicular triangle resulting in upper extremity symptoms such as pain and swelling without thrombosis (6). 


\section{Anatomy and pathophysiology}

Anatomically, thoracic outlet consists of three different spaces, the costoclavicular space, interscalene triangle, and the subcoracoid space $(6,7)$. Venous compression most commonly occurs within the costoclavicular space or cervicoaxillary canal (6). The costoclavicular space is the space confined by $1^{\text {st }}$ rib inferiorly, subclavius muscle and clavicle superiorly, anterior scalene muscle laterally and subclavius muscle/costoclavicular ligament medially $(6,8)$.

In cadaveric studies, abnormal anterior displacement of the anterior scalene muscle insertion site on the first rib has been described in about $15 \%$ of the normal population resulting in anterior and inferior displacement of the subclavian vein and artery and secondary compression of the subclavian vein at the costoclavicular angle (9). Hypertrophy of the scalene muscle and tendon or costoclavicular ligament are among other factors that can contribute to vTOS $(10,11)$.

Several other anatomical abnormalities have been described in literature such as rib anomalies (cervical rib, rudimentary rib or fused first and second ribs), abnormal course of subclavian artery in relation to anterior scalene muscle, fibrosis or congenital bands but most of these are more strongly associated with arterial TOS $(6,8,12)$. In a recent retrospective study, osseous abnormalities were present in $29 \%$ of their cohort of 400 patients (most commonly cervical ribs, about $70 \%$ of all osseous abnormalities) and this was associated with high likelihood of arterial TOS and low likelihood of vTOS (12).

Irrespective of the exact underlying etiology in vTOS, compression results in stasis and increased venous pressure in the affected upper extremity. Combination of blood stasis with endothelial injury to the axillosubclavian vein from repetitive trauma of upper extremity provocative movements such as shoulder abduction eventually leads to venous thrombosis (10). Presenting symptoms in these patients include arm swelling from decreased venous drainage, pain, cyanosis and coldness and can rarely cause limb threatening ischemia (phlegmasia) (2). In chronic cases these symptoms may be absent or have resolved over time secondary to development of sufficient collateral flow and these collateral veins might be visible in the neck and upper chest.

\section{Epidemiology}

vTOS is the second most common type of TOS comprising about $3-4 \%$ of all TOS patients. Neurogenic TOS is the most common type (95\% of the TOS patients) and arterial TOS is least common (1-2\% of the TOS patients). The overall incidence of vTOS has been reported between $1 / 100,000$ to $11 / 100,000$ patients per year $(10,11,13)$. However, the true prevalence of the disorder is hard to estimate since it represents a spectrum and symptomatic patients are only a small proportion with the underlying anatomical predisposition. For instance, abnormal axillosubclavian vein compression has been reported to be present in about $10 \%$ of normal population detected by ultrasound screening (14).

vTOS patients are usually young otherwise healthy individuals in their 30s (11). vTOS is more frequent in males with a male to female ratio of 2:1. This is in contrast to TOS overall, which is more frequent among females $(11,15,16)$. In up to $70 \%$ of cases, vTOS affects the dominant upper extremity (usually the right arm) and occurs after excessive shoulder abduction or unusual activity (2).

\section{Diagnosis}

The role of imaging for the management of vTOS can be divided in to four main categories. First, to confirm or supplement the diagnosis. Second, to potentially identify the cause of compression. Third, to diagnose associated complications of vTOS such as venous thrombosis or development of venous collaterals and lastly, for long term surveillance of the patients and detection of thrombosis recurrence.

\section{Chest radiograph}

It can be argued that initial step for the work up of TOS patients after obtaining a detailed history and physical examination, irrespective of the type of compression symptoms, is a frontal chest radiograph $(17,18)$. Obtaining a radiograph as the initial step can help diagnose presence of a cervical rib, first rib abnormalities, abnormal elongation of the $\mathrm{C} 7$ transverse, as well as degenerative or posttraumatic osseous changes in the region of interest $(17,18)$. Additionally, chest radiographs may potentially reveal large neoplasms close to the region such as apical lung cancers (19). Despite the diagnostic value of frontal chest radiograph, previous studies have demonstrated that osseous abnormalities have weak correlation with vTOS and are more correlated with arterial TOS (12). Additionally, chest radiograph has very low sensitivity and should be used for screening purposes (19). 

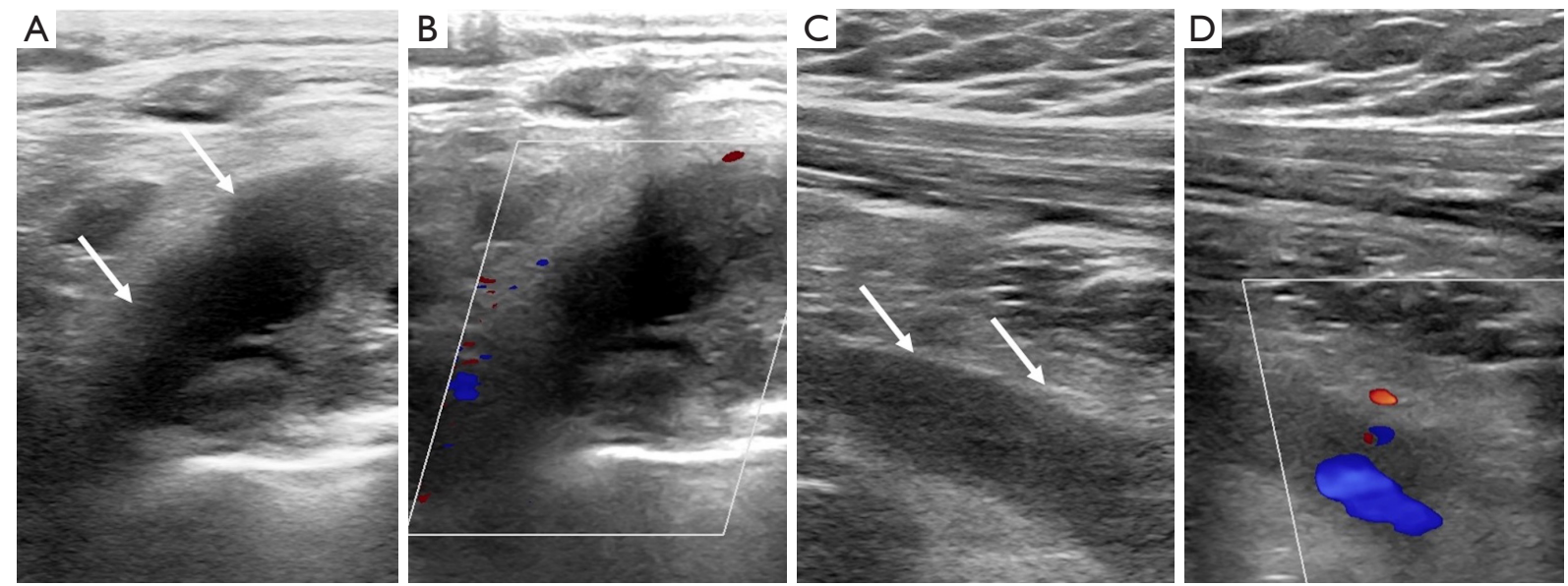

Figure 1 Grayscale and color Doppler images of a patient presenting with left upper extremity pain and swelling for 4 weeks. Ultrasound images show hypoechoic thrombus in the left subclavian (A, white arrows) and axillary veins (B, white arrows). Corresponding color Doppler images show lack of flow in keeping with thrombosis (C,D).

\section{Duplex ultrasound (DUS)}

In patients suffering from PSS or effort thrombosis, or in any patient with suspicion for deep vein thrombosis (DVT), DUS is the initial test of choice (Figure 1). It is relatively cheap, widely available, and lacks ionizing radiation. DUS has a reported sensitivity and specificity of $78-100 \%$ and $82-100 \%$, respectively, for the detection of venous thrombosis (20). One of the biggest advantages of the DUS for evaluating vTOS patients is the dynamic nature of the study, which allows evaluation of the venous flow alterations (or venous occlusion) during arm movements such as shoulder abduction but the significance of these findings remains unknown $(11,16,19,21)$. There are also several limitations associated with application of DUS for the diagnosis of vTOS. Ultrasound may not detect some of the rare but serious underlying etiologies such as lung tumors. Shadowing resulting from osseous structures within the costoclavicular space affects the visibility of venous structures and limits performance of compression maneuvers on the axillosubclavian veins (22).

\section{Dynamic contrast-enbanced CT and MRI}

CT and MRI venography are excellent imaging tools that can evaluate the extent of thrombosis, and collateral development, rule out other diagnoses such as adjacent tumors, and help diagnose the area of narrowing and associated anatomical abnormalities $(21,22)$. Both CT and MR venography can be performed with and without provocative maneuvers, most commonly elevation of the arms above the head, to further evaluate and narrow down the etiology and identify the exact area of narrowing or to diagnose subtle cases. At our institution, the CT venography imaging protocol consists of imaging patients after injection of 75-90 $\mathrm{mL}$ of intravenous contrast material through the contralateral arm to the symptomatic side followed by imaging with arms in adduction at 60-70 s delay and repeating the CT acquisition with arms above the head (Figure 2). The data on CT venograms is limited but in a study performed by Gillet et al. subclavian artery narrowing more than $50 \%$ on dynamic CT was associated with symptomatic TOS (23).

\section{Catheter-directed venography}

Catheter-directed venography is considered the gold standard for diagnosing vTOS. It allows for accurate evaluation of venous luminal narrowing, presence of thrombus, chronicity of the occlusion and degree of collateralization. Venography can be repeated during provocative maneuvers as well to evaluate the degree of external compression. It is also performed when catheterdirected thrombolysis (CDT) or other interventions are planned $(22,24)$.

\section{Treatment}

The treatment strategy for vTOS depends on different factors including the severity of symptoms, presence of 

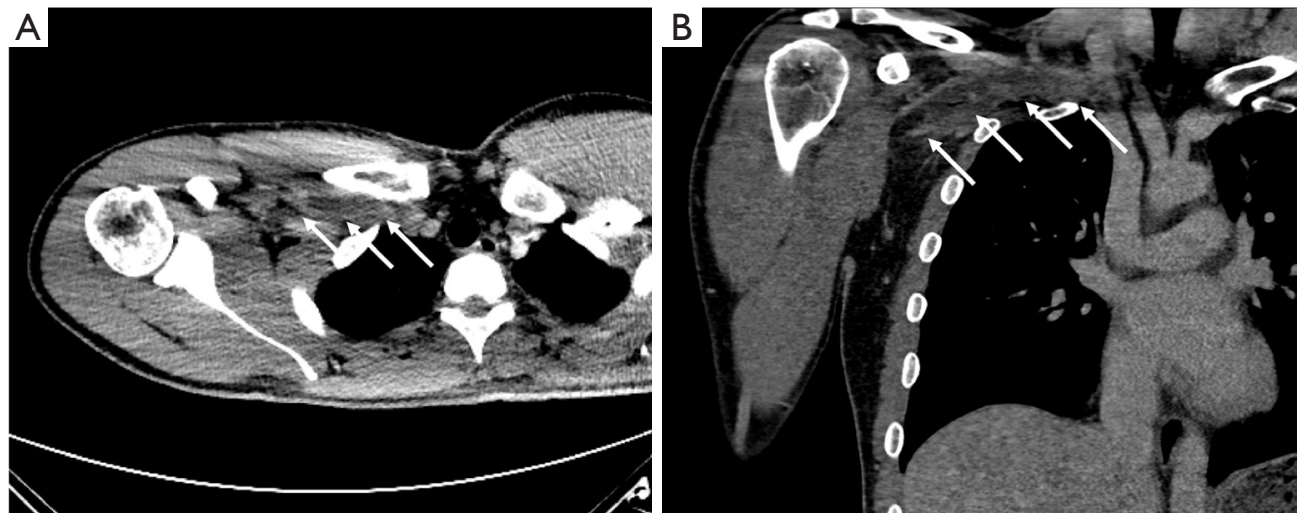

Figure 2 CT images in venous phase in sagittal (A) and coronal planes (B) show acute thrombosis in the axillary and subclavian veins (white arrows).

thrombosis, and chronicity of the condition. Irrespective of the treatment chosen, the ultimate goal is first to relieve the initial upper extremity pain and swelling as well as avoiding pulmonary embolism. Secondary goals include, treat the cause of compression to minimize the chance of recurrence, avoid chronic symptoms or post thrombotic syndrome and most importantly, restore the function of affected upper extremity to the fullest (25).

\section{Medical management}

The initial step in the management of all patients diagnosed with upper extremity DVT in the absence of increased bleeding risk is anticoagulation. The American College of Chest Physicians recommends at least 3 months of anticoagulation for patients diagnosed with upper extremity DVT (26). Although, hypercoagulable states such as protein $\mathrm{C}, \mathrm{S}$, and antithrombin-III deficiencies, lupus anticoagulants and antiphospholipid antibodies, factor $\mathrm{V}$ Leiden and prothrombin gene G20210A mutation are associated with upper extremity DVT (27-31), their association with PSS is less known. Therefore, there is no consensus among experts for routine work up in the PSS patients (32).

The choice of anticoagulation depends on the type of interventions planned for each individual patient. Patients presenting with acute/subacute axillosubclavian thrombosis who will benefit from CDT and surgical interventions could be started on therapeutic unfractionated heparin (goal PTT of 1.5-2.5 times reference) so it could be modified periprocedurally. For example, the dose of heparin should be lowered at the time of CDT to decrease the risk of bleeding $(33,34)$. At our institution, we lower the dose of unfractionated heparin to 500 units/hour at the time of thrombolysis without adjustment for PTT in adults. Once the catheter based and surgical interventions are completed, patients can be bridged to long term therapy prior to discharge. The length of treatment after successful thrombolysis and thoracic outlet decompression is also debatable but most experts recommend a period of at least 3 months and extending this interval in patients with recurrent thrombosis. However, patients with residual venous scarring following thoracic outlet decompression would benefit from anticoagulation with or without percutaneous venoplasty since anticoagulation would keep the treated vein patent, decrease the chance of thrombosis and might result in recanalization of the occluded segments.

Symptomatic treatment including arm elevation, use of compression stockings and pain relief with non-steroidal anti-inflammatory medications should be considered in the acute phase of PSS and for patients suffering from postthrombotic syndrome.

\section{$C D T$}

CDT has a pivotal role in the management of vTOS and PSS. The goal of CDT is to dissolve the clot and restore a patent venous lumen. Successful CDT decreases the occurrence of post thrombotic syndrome, venous scarring and also helps to relieve acute symptoms (35-37).

When it comes to successful lysis of the axillosubclavian thrombus, early diagnosis and intervention is key. In the acute/subacute stages (within the first two weeks), CDT has been reported to be highly successful and could result in near complete clot resolution in up to $80 \%$ of the patients $(38,39)$. The rate of successful thrombolysis drops rapidly after this period as the clot gets organized. In a study 

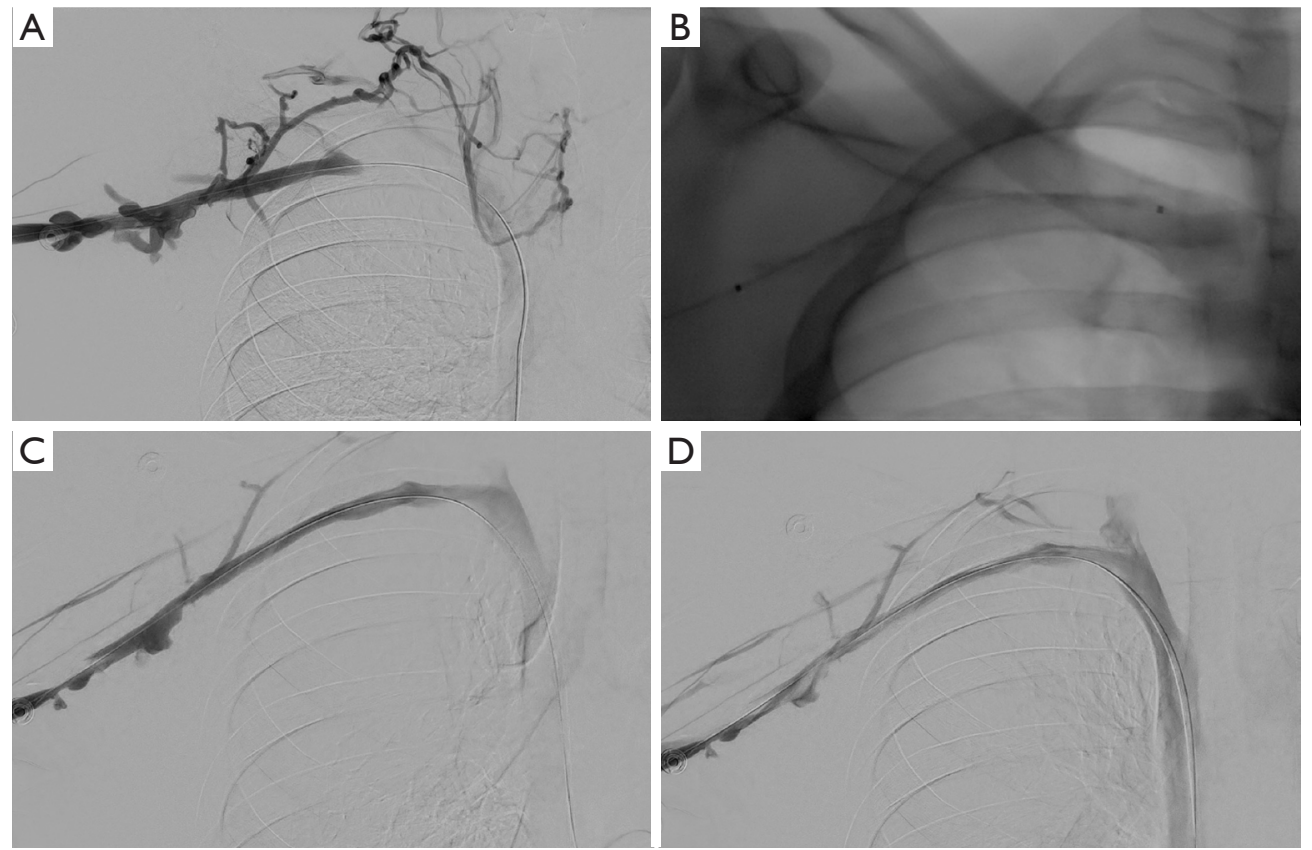

Figure 3 Patient presenting with right upper extremity pain and swelling. Catheter directed venogram shows complete occlusion of the subclavian vein with mild collateralization of the flow (A). A multisidehole infusion catheter was placed across the occlusion and thrombolysis was performed overnight (B). C and D show completion venogram after successful thrombolysis with marked resolution of the clot burden and patency of the subclavian vein.

performed by Molina et al., CDT was only successful in about $30 \%$ of the patients who underwent thrombolysis in 2-12 weeks after initial symptoms (40).

At our institution, the procedure is performed under moderate sedation (general anesthesia only in select cases) using intravenous administration of midazolam and fentanyl. Venous access is usually obtained in the upper arm of the affected side under real time ultrasound and a 21 micro-puncture access set. It is very important to limit the vascular trauma when accessing the vein to avoid access site complications such as bleeding or hematoma during the lysis. After insertion of appropriately sized vascular sheath (either 6 or $7 \mathrm{Fr}$ ) and diagnostic venography for mapping the venous anatomy and extent of occlusion, the thrombosis is crossed with any combination of hydrophilic wires and catheter. A multisidehole infusion catheter (i.e., CraggMcNamara catheter (Medtronic, Dublin, Ireland)) is then selected based on the length of the thrombosed area and laid across the occlusion. Alteplase is then infused through the infusion catheter overnight at usually $1 \mathrm{mg}$ /hour or $0.01 \mathrm{mg} / \mathrm{kg} /$ hour and heparin is infused at a subtherapeutic dose either through the vascular sheath or a peripheral intravenous catheter. Patients are observed overnight at intensive care unit with repeat invasive venography performed the following day to monitor the treatment progression. Lysis can be continued for 24-48 hours until satisfactory results are obtained (Figure 3).

Pharmacomechanical thrombectomy using devices such as Angiojet (Boston Scientific, Marlborough, MA) or EKOS (BTG, London, UK) can be used as alternative or augmentative tool to infusion catheters. Angiojet enables the operator to perform single session thrombolysis and clot removal using alteplase pulse spray into the clot followed by thrombectomy. EKOS uses acoustic pulsation in combination with alteplase infusion to enhance the catheter directed thrombolysis. It should be considered that there is very limited or no evidence in the utility or superiority of these devices in upper extremity DVT over infusion catheters.

Once maximum removal of clot is achieved by pharmachological thrombolysis or pharmacomechanical thrombectomy, adjunct transluminal venous reconstruction techniques such as venous angioplasty should be considered to treat the residual scarring from thrombosis and establish adequate lumen for blood flow (41) (Figure 4). Another technique that might be helpful at this stage to evaluate for 

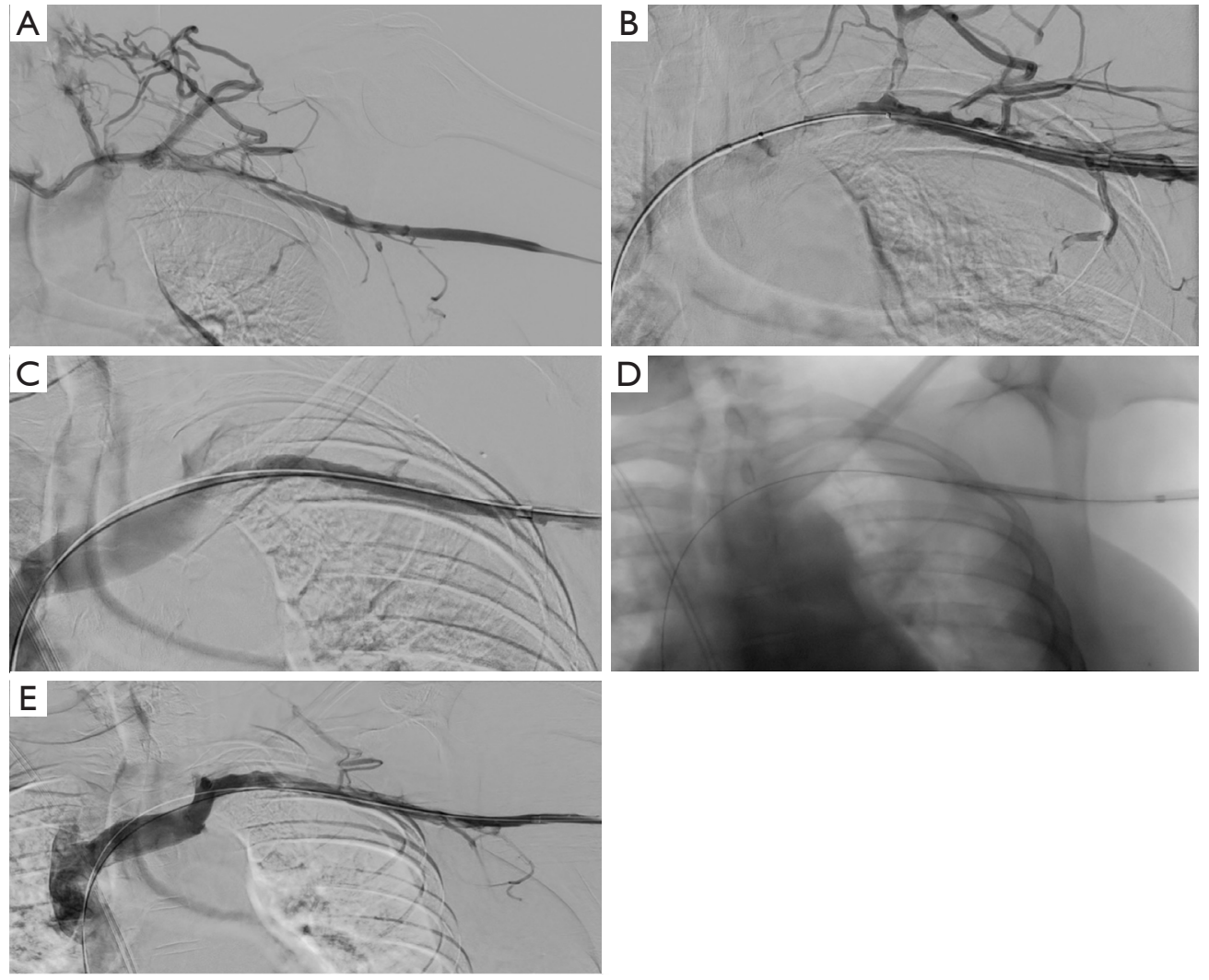

Figure 4 Catheter directed venography in a patient with PSS shows occlusion of the subclavian vein and narrowing of the axillary vein with marked collateralization of the flow (A). After crossing the area of occlusion (B) and angioplasty, repeat venogram (C) shows improved flow with moderate residual scarring and chronic thrombus. After further angioplasty (D), completion venogram (E) shows marked improvement of the luminal narrowing and good flow.

external compression is intravascular ultrasound (IVUS). IVUS has been shown to be very helpful in patients with left common iliac vein compression (May-Thurner) syndrome to make the diagnosis, determine the cause of compression and asses for residual clot (42).

\section{Surgical decompression}

Thoracic outlet decompression has traditionally been and remains one the most important components of vTOS and PSS management. Lack of adequate decompression will result in poor symptomatic relief, lower patency rates and higher recurrence of thrombosis (43). Although there is no consensus about the exact surgical method secondary to lack of randomized data, most algorithms include first rib resection as the most important component of surgical decompression for vTOS, debulking of the subclavius and anterior scalene and venolysis (41). The timing of surgery after thrombolysis also varies among different treatment algorithms. For example some physicians favor to wait for 1-3 months to allow for endothelial healing after successful thrombolysis prior to surgical decompression (44). However, it is currently accepted that it is safe to perform the thoracic outlet decompression during the same admission for thrombolysis and there is a trend towards shortening the interval between CDT and surgical decompression to lower the risk of recurrence.

Following decompression, many patients will have residual venous scarring and chronic thrombus. There are several surgical and percutaneous approaches including intraoperative venolysis and patch venoplasty during decompressive surgery with excellent outcomes and reported $100 \%$ patency following these procedures (40). Other approach is anticoagulating these patients with or without percutaneous angioplasty (delayed or early) (11).

Another option for patients with residual venous scarring 

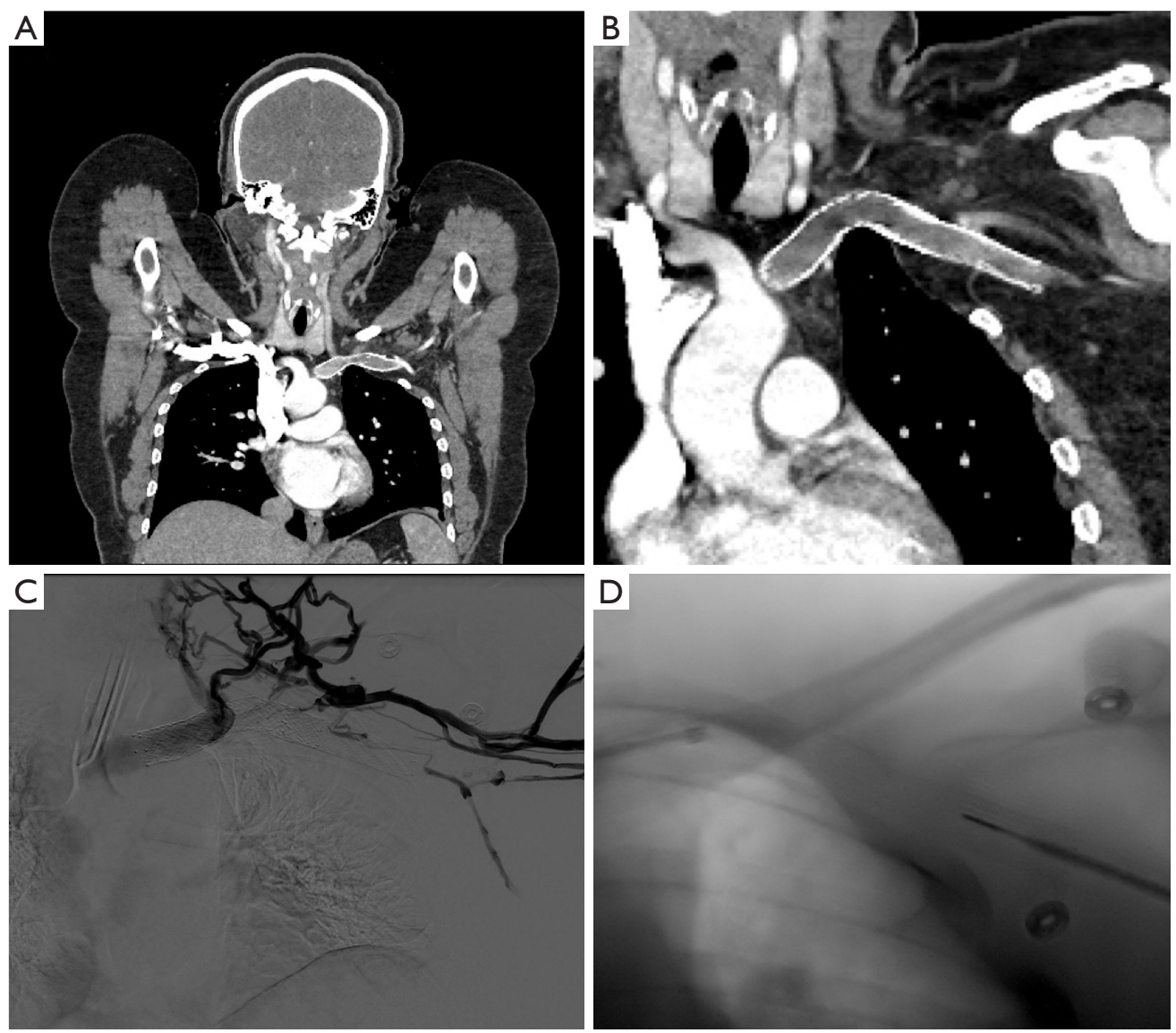

Figure 5 CT venogram with arm in abduction show complete occlusion, narrowing and irregularity of the stent across clavicle in the central portion of the subclavian vein $(\mathrm{A}, \mathrm{B})$ in a patient after catheter directed thrombolysis and first rib resection with residual venous narrowing unresponsive to angioplasty. Patient underwent stenting at another institution and presented with worsening symptoms after 2 years. Venogram shows complete occlusion of the stent $(\mathrm{C})$ and attempts at crossing the area of occlusion, even using sharp recanalization were unsuccessful (D).

non-responsive to angioplasty alone is stent placement. In a study performed by Rajendran et al. patients with residual scarring and stenosis after thrombolysis, surgical decompression and venolysis underwent angioplasty and primary stent placement with dedicated venous stents without any major complications and primary and secondary patency of $55 \%$ and $100 \%$ at 2 years, respectively (5). Although, stent outcomes reported in the study are very good, stenting of the subclavian vein poses risk of stent fracture because of repetitive movement in this location and recurrent occlusion (Figure 5). Because the patient being affected by PSS is usually young and active, this adds to the importance of this potential complication. Since the long term patency of subclavian stents is unknown, in authors opinion, stenting in this location should only be considered in select patients and after exhaustion of every other option such as venolysis and patch venoplasty.

\section{Conclusions}

PSS or effort thrombosis is a part of vTOS spectrum that mostly affects young otherwise healthy individuals. All current algorithms for the management of PSS require a multidisciplinary approach with anticoagulation and CDT as the initial treatment. Early diagnosis and aggressive early intervention with CDT and decompressive surgery increase the likelihood of complete resolution of acute symptoms and full restoration of limb function.

\section{Acknowledgments}

Funding: None. 


\section{Footnote}

Provenance and Peer Review: This article was commissioned by the editorial office, Cardiovascular Diagnosis and Therapy for the series "Compressive Vascular Syndromes". The article has undergone external peer review.

Conflicts of Interest: The authors have completed the ICMJE uniform disclosure form (available at http://dx.doi. org/10.21037/cdt-20-168). The series “Compressive Vascular Syndromes" was commissioned by the editorial office without any funding or sponsorship. Dr. Kolber served as the unpaid Guest Editor of the series. The authors have no other conflicts of interest to declare.

Ethical Statement: The authors are accountable for all aspects of the work in ensuring that questions related to the accuracy or integrity of any part of the work are appropriately investigated and resolved.

Open Access Statement: This is an Open Access article distributed in accordance with the Creative Commons Attribution-NonCommercial-NoDerivs 4.0 International License (CC BY-NC-ND 4.0), which permits the noncommercial replication and distribution of the article with the strict proviso that no changes or edits are made and the original work is properly cited (including links to both the formal publication through the relevant DOI and the license). See: https://creativecommons.org/licenses/by-nc-nd/4.0/.

\section{References}

1. Horattas MC, Wright DJ, Fenton AH, et al. Changing concepts of deep venous thrombosis of the upper extremity--report of a series and review of the literature. Surgery 1988;104:561-7.

2. Sharafuddin MJ, Sun S, Hoballah JJ. Endovascular management of venous thrombotic diseases of the upper torso and extremities. J Vasc Interv Radiol 2002;13:975-90.

3. Aziz S, Straehley CJ, Whelan TJ Jr. Effort-related axillosubclavian vein thrombosis. A new theory of pathogenesis and a plea for direct surgical intervention. Am J Surg 1986;152:57-61.

4. Hughes ES. Venous obstruction in the upper extremity; Paget-Schroetter's syndrome; a review of 320 cases. Surg Gynecol Obstet 1949;88:89-127.

5. Rajendran S, Cai TY, Loa J, et al. Early outcomes using dedicated venous stents in the upper limb of patients with venous thoracic outlet syndrome: A single centre experience. CVIR Endovascular 2019;2:22.

6. Klaassen Z, Sorenson E, Tubbs RS, et al. Thoracic outlet syndrome: a neurological and vascular disorder. Clin Anat 2014;27:724-32.

7. Charon JP, Milne W, Sheppard DG, et al. Evaluation of MR angiographic technique in the assessment of thoracic outlet syndrome. Clin Radiol 2004;59:588-95.

8. Freischlag J, Orion K. Understanding thoracic outlet syndrome. Scientifica (Cairo) 2014;2014:248163.

9. Wayman J, Miller S, Shanahan D. Anatomical variation of the insertion of scalenus anterior in adult human subjects: implications for clinical practice. J Anat 1993;183:165-7.

10. Butros SR, Liu R, Oliveira GR, et al. Venous compression syndromes: clinical features, imaging findings and management. Br J Radiol 2013;86:20130284.

11. Moore R, Wei Lum Y. Venous thoracic outlet syndrome. Vasc Med 2015;20:182-9.

12. Weber AE, Criado E. Relevance of bone anomalies in patients with thoracic outlet syndrome. Ann Vasc Surg 2014;28:924-32.

13. Edwards DP, Mulkern E, Raja AN, et al. Trans-axillary first rib excision for thoracic outlet syndrome. J R Coll Surg Edinb 1999;44:362-5.

14. Longley DG, Yedlicka JW, Molina EJ, et al. Thoracic outlet syndrome: evaluation of the subclavian vessels by color duplex sonography. AJR Am J Roentgenol 1992;158:623-30.

15. Kellar J, Trigger C. Thoracic outlet syndrome with secondary Paget Schroetter Syndrome: a rare case of effort-induced thrombosis of the upper extremity. West J Emerg Med 2014;15:364-5.

16. Moriarty JM, Bandyk DF, Broderick DF, et al. ACR Appropriateness Criteria Imaging in the Diagnosis of Thoracic Outlet Syndrome. J Am Coll Radiol 2015;12:438-43.

17. Gillard J, Perez-Cousin M, Hachulla E, et al. Diagnosing thoracic outlet syndrome: contribution of provocative tests, ultrasonography, electrophysiology, and helical computed tomography in 48 patients. Joint Bone Spine 2001;68:416-24.

18. Illig KA, Donahue D, Duncan A, et al. Reporting standards of the Society for Vascular Surgery for thoracic outlet syndrome. J Vasc Surg 2016;64:e23-35.

19. Zucker EJ, Ganguli S, Ghoshhajra BB, et al. Imaging of venous compression syndromes. Cardiovasc Diagn Ther 2016;6:519-32.

20. Chin EE, Zimmerman PT, Grant EG. Sonographic evaluation of upper extremity deep venous thrombosis. J Ultrasound Med 2005;24:829-38; quiz 39-40. 
21. Demondion $X$, Herbinet $P$, Van Sint Jan S, et al. Imaging assessment of thoracic outlet syndrome. Radiographics 2006;26:1735-50.

22. Povlsen S, Povlsen B. Diagnosing Thoracic Outlet Syndrome: Current Approaches and Future Directions. Diagnostics (Basel) 2018;8:21.

23. Gillet R, Teixeira P, Meyer JB, et al. Dynamic CT angiography for the diagnosis of patients with thoracic outlet syndrome: Correlation with patient symptoms. J Cardiovasc Comput Tomogr 2018;12:158-65.

24. Matsumura JS, Rilling WS, Pearce WH, et al. Helical computed tomography of the normal thoracic outlet. J Vasc Surg 1997;26:776-83.

25. Thompson RW. Comprehensive management of subclavian vein effort thrombosis. Semin Intervent Radiol 2012;29:44-51.

26. Kearon C, Akl EA, Ornelas J, et al. Antithrombotic Therapy for VTE Disease: CHEST Guideline and Expert Panel Report. Chest 2016;149:315-52.

27. Ellis MH, Manor Y, Witz M. Risk factors and management of patients with upper limb deep vein thrombosis. Chest 2000;117:43-6.

28. Seligsohn U, Lubetsky A. Genetic susceptibility to venous thrombosis. N Engl J Med 2001;344:1222-31.

29. Leebeek FW, Stadhouders NA, van Stein D, et al. Hypercoagulability states in upper-extremity deep venous thrombosis. Am J Hematol 2001;67:15-9.

30. Héron E, Lozinguez O, Alhenc-Gelas M, et al. Hypercoagulable states in primary upper-extremity deep vein thrombosis. Arch Intern Med 2000;160:382-6.

31. De Cicco M, Matovic M, Balestreri L, et al. Antithrombin III deficiency as a risk factor for catheter-related central vein thrombosis in cancer patients. Thromb Res 1995;78:127-37.

32. Hangge P, Rotellini-Coltvet L, Deipolyi AR, et al. PagetSchroetter syndrome: treatment of venous thrombosis and outcomes. Cardiovasc Diagn Ther 2017;7:S285-90.

33. Enden T, Sandvik L, Klow NE, et al. Catheterdirected Venous Thrombolysis in acute iliofemoral vein thrombosis--the CaVenT study: rationale and design of a multicenter, randomized, controlled, clinical trial (NCT00251771). Am Heart J 2007;154:808-14.
34. Schulman S, Beyth RJ, Kearon C, et al. Hemorrhagic complications of anticoagulant and thrombolytic treatment: American College of Chest Physicians Evidence-Based Clinical Practice Guidelines (8th Edition). Chest 2008;133:257S-98S.

35. Molina JE, Hunter DW, Dietz CA. Protocols for Paget-Schroetter syndrome and late treatment of chronic subclavian vein obstruction. Ann Thorac Surg 2009;87:416-22.

36. Vik A, Holme PA, Singh K, et al. Catheter-directed thrombolysis for treatment of deep venous thrombosis in the upper extremities. Cardiovasc Intervent Radiol 2009;32:980-7.

37. Alesh I, Kayali F, Stein PD. Catheter-directed thrombolysis (intrathrombus injection) in treatment of deep venous thrombosis: a systematic review. Catheter Cardiovasc Interv 2007;70:143-8.

38. Doyle A, Wolford HY, Davies MG, et al. Management of effort thrombosis of the subclavian vein: today's treatment. Ann Vasc Surg 2007;21:723-9.

39. Adelman MA, Stone DH, Riles TS, et al. A multidisciplinary approach to the treatment of PagetSchroetter syndrome. Ann Vasc Surg 1997;11:149-54.

40. Molina JE, Hunter DW, Dietz CA. Paget-Schroetter syndrome treated with thrombolytics and immediate surgery. J Vasc Surg 2007;45:328-34.

41. Carlon TA, Sudheendra D. Interventional Therapy for Upper Extremity Deep Vein Thrombosis. Semin Intervent Radiol 2017;34:54-60.

42. Forauer AR, Gemmete JJ, Dasika NL, et al. Intravascular ultrasound in the diagnosis and treatment of iliac vein compression (May-Thurner) syndrome. J Vasc Interv Radiol 2002;13:523-7.

43. Lugo J, Tanious A, Armstrong P, et al. Acute PagetSchroetter syndrome: does the first rib routinely need to be removed after thrombolysis? Ann Vasc Surg 2015;29:1073-7.

44. Machleder HI. Evaluation of a new treatment strategy for Paget-Schroetter syndrome: spontaneous thrombosis of the axillary-subclavian vein. J Vasc Surg 1993;17:305-15; discussion 316-7.
Cite this article as: Habibollahi P, Zhang D, Kolber MK, Pillai AK. Venous thoracic outlet syndrome. Cardiovasc Diagn Ther 2021;11(5):1150-1158. doi: 10.21037/cdt-20-168 